\title{
REAÇÕES DE POLIMERIZAÇÃO VIA METÁTESE DE DIENO ACÍCLICO (ADMET) EM MINIEMULSÃO
}

\author{
P. B. CARDOSO ${ }^{1,2,3}$, A. MUSYANOVICH ${ }^{2}$, K. LANDFESTER ${ }^{2}$, C. SAYER ${ }^{1}$, P. H. H. ARAÚJO ${ }^{1}$, \\ M. A. R. MEIER ${ }^{3}$ \\ ${ }^{1}$ Universidade Federal de Santa Catarina, Departamento de Engenharia Química e Engenharia de \\ Alimentos, Florianópolis, Brasil \\ ${ }^{2}$ Max Planck Institute for Polymer Research, Mainz, Germany \\ ${ }^{3}$ Institute of Organic Chemistry, Karlsruhe Institute of Technology, Karlsruhe, Germany \\ E-mail para contato: pri.barretocardoso@gmail.com
}

\begin{abstract}
RESUMO - A utilização de óleos vegetais como matéria-prima renovável na indústria de polímeros é um desafio e uma oportunidade que pode permitir a substituição dos compostos oriundos do petróleo. Neste contexto, a metátese de olefinas aparece como técnica promissora para a obtenção de polímeros provenientes de óleos vegetais através de modificações nas porções olefínicas (duplas ligações) dos ácidos graxos. Além da possibilidade da ausência de solventes orgânicos, caracterizando-se como um sistema ambientalmente amigável, as reações em miniemulsão permitem a obtenção de nanopartículas com características únicas e de grande interesse comercial, como nanopartículas ocas, híbridas, nanocápsulas, entre outros. O presente trabalho descreve a aplicação de reações de polimerização do tipo ADMET (acyclic diene metathesis) em miniemulsão. Foram obtidos polímeros com massa molar $\left(\mathrm{M}_{\mathrm{n}}\right)$ de até $15 \mathrm{kDa}$, dependendo dos tipos de catalisador e surfactante (aniônio, catiônico ou não iônico) utilizados.
\end{abstract}

\section{INTRODUÇÃO}

Os óleos vegetais representam um grande potencial como fonte alternativa para a produção de materiais poliméricos, pois além de serem oriundos de fontes renováveis, estão disponíveis em todo o mundo, apresentam preços relativamente baixos e possibilidades de aplicações com alto valor agregado. Essas vantagens tornam os óleos vegetais atraentes e viáveis para a indústria de polímeros, oferecendo uma oportunidade para a substituição dos processos de polimerização existentes baseados em compostos provenientes do petróleo (Montero de Espinosa e Meier, 2011; Cardoso et al., 2014). Como grande parte dos ácidos graxos e seus derivados apresentam ligações carbono-carbono insaturadas em sua estrutura molecular, a metátese de olefinas aparece como técnica promissora para a obtenção de polímeros com altas massas molares provenientes dessas matérias-primas renováveis, contribuindo para a obtenção de novos monômeros e polímeros através de reações de polimerização mais eficientes e ampliando as possibilidades de aplicação dos óleos vegetais (Meier et al., 2007).

Nos últimos anos, a técnica de polimerização em miniemulsão tem ganhado destaque em termos de publicações e desenvolvimento de materiais poliméricos inovadores e mais acessíveis, uma vez 


\section{9 a 22 de outubro de 2014 \\ Florianópolis/SC}

que a técnica combina muitas características atraentes da polimerização em emulsão convencional (ausência de solventes, maior facilidade de controle de temperatura e compartimentalização dos radicais nas partículas de polímero, o que permite obter altas velocidades de polimerização e polímeros de elevada massa molar) com a possibilidade de usar reagentes ou incorporar compostos insolúveis em água (Asua, 2002; Antonietti e Landfester, 2002), tais como diversos óleos vegetais (Cardoso et al., 2013). Além disso, tais processos permitem a produção de nanopartículas com características únicas e de grande interesse comercial, destacando-se a obtenção de nanopartículas estruturadas (ocas, híbridas, com microestrutura bem definida), a encapsulação de sólidos inorgânicos e de princípios ativos, entre outros (Landfester, 2009; Staudt et al., 2013).

Há um crescente interesse na realização de reações de metátese em fase aquosa com o objetivo de trabalhar com sistemas ambientalmente amigáveis, mas poucos trabalhos de reações de metátese em miniemulsão foram publicados (Lynn et al.,1996; Pecher e Mecking, 2007; 2010). Neste contexto, o presente trabalho tem como objetivo estudar a polimerização de monômeros derivados de óleos vegetais através de reações ADMET em miniemulsão.

\section{MATERIAIS E MÉTODOS}

\subsection{Materiais}

Ácido 10-undecenóico, 1,3-propanodiol, ácido p-toluenosulfônico e tolueno foram utilizados na reação de preparo do monômero. Para a purificação, óxido de alumínio básico, sílica gel, sulfato de magnésio anidro, hexano e éter dietílico foram usados. Dodecil sulfato de sódio (SDS), brometo de cetiltrimetilamônio (CTAB) e Lutensol AT80, um álcool graxo etoxilado com 80 unidades de óxido de etileno (massa molar de 3,8 kDa), foram utilizados como surfactante. Os catalisadores usados foram Hoveyda-Grubbs de $2^{\mathrm{a}}$ geração (HG2), Grubbs de $1^{\mathrm{a}}$ geração (G1) e Umicore M2 (UM2). Água destilada foi utilizada como meio contínuo das miniemulsões. Todos os materiais foram usados como recebidos.

\subsection{Preparo do Monômero}

Ácido 10-Undecenóico (50,0 g, 0,27 mol), 1,3-propanodiol (8,4 g, 0,11 mol) e ácido ptoluenossulfônico $(3,0 \mathrm{~g}, 0,0157 \mathrm{~mol})$ foram adicionados a um balão volumétrico equipado com agitação magnética, aquecimento e aparato Dean-Stark. Tolueno $(200 \mathrm{~mL})$ foi adicionado como solvente e a mistura foi então aquecida até o refluxo. Água foi coletada como subproduto e, após o término da reação, a mistura foi resfriada. Para a purificação do monômero, o tolueno foi removido com o auxílio de um rotaevaporador e o resíduo foi filtrado através de uma coluna de óxido de alumínio básico, usando hexano como eluente. Após a remoção do hexano, o produto é dissolvido em éter dietílico $(200 \mathrm{~mL})$ e lavado duas vezes com água $(200 \mathrm{~mL})$. A fração orgânica foi seca com sulfato de magnésio anidro e o solvente foi então removido. O produto desejado foi isolado em $78 \%$ (35,2 $\mathrm{g})$ de rendimento e analisado por RMN.

${ }^{1} \mathrm{H} \mathrm{RMN}\left(\mathrm{CDCl}_{3}\right): \delta=5.85-5.76\left(\mathrm{~m}, 2 \mathrm{H}, 2 \times-\mathrm{CH}=\mathrm{CH}_{2}\right), 5.00-4.91\left(\mathrm{~m}, 4 \mathrm{H}, 2 \times \mathrm{CH}=\mathrm{CH}_{2}\right)$,

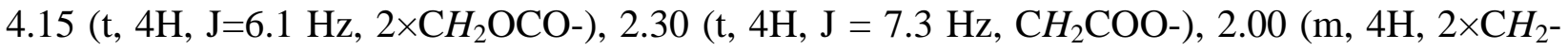
$\left.\mathrm{CH}=\mathrm{CH}_{2}\right), 1.99-1.94\left(\mathrm{~m}, 2 \mathrm{H}, \mathrm{J}=6.1 \mathrm{~Hz}, \mathrm{CH}_{2} \mathrm{CH}_{2} \mathrm{OCO}-\right), 1.64-1.58\left(\mathrm{~m}, 4 \mathrm{H}, 2 \times \mathrm{CH}_{2} \mathrm{CH}_{2} \mathrm{COO}-\right)$, 
1.38-1.34 (m, 4H, 2×CH $)$ 1.29-1.24 (br.s, $\left.16 \mathrm{H}, 2 \times\left[4 \mathrm{CH}_{2}\right]\right)$ ppm. ${ }^{13} \mathrm{C} \mathrm{RMN}\left(\mathrm{CDCl}_{3}\right): \delta=173.6$ (s, -COO-), 139.0 (s, $\left.-\mathrm{CH}=\mathrm{CH}_{2}\right), 114.1$ (s, $\left.-\mathrm{CH}=\mathrm{CH}_{2}\right), 60.7$ (s, $\left.\mathrm{CH}_{2} \mathrm{OCO}-\right), 34.1$ (s, $\left.\mathrm{CH}_{2}\right), 33.7$ (s, $\mathrm{CH}_{2}$ ), 29.2 (s, $\left.C \mathrm{H}_{2}\right), 29.1\left(\mathrm{~s}, \mathrm{CH}_{2}\right), 29.0\left(\mathrm{~s}, \mathrm{CH}_{2}\right), 28.8\left(\mathrm{~s}, \mathrm{CH}_{2}\right), 24.8\left(\mathrm{~s}, C \mathrm{H}_{2}\right) \mathrm{ppm}$. MS (EI): $\mathrm{m} / z$ $=408[\mathrm{M}]^{+}$, calc. 408.3239 .

\subsection{Reações ADMET}

Reações de polimerização em massa e em miniemulsão via ADMET do monômero 1,3propileno diundec-10-enoato foram realizadas. Para as reações em miniemulsão, inicialmente, duas pré-emulsões foram preparadas; a primeira (1) misturando água (2 mL) e surfactante com clorofórmio $(0,25 \mathrm{~g})$ e catalisador e a segunda (2) misturando água $(8 \mathrm{~mL})$ e surfactante com o monômero (1 g). Após 20 minutos de agitação, 1 e 2 foram sonicadas separadamente (Branson Ultrasonics Corp., Branson Digital Sonifier W450) durante 1 minuto com uma amplitude de 60\% e, então, misturadas e sonicadas novamente por mais dois minutos. Banho de gelo foi utilizado para evitar o aumento de temperatura durante a sonicação. Um esquema simplificado da condução das reações ADMET em miniemulsão é apresentado na Figura 1. Quando reações em massa foram realizadas, monômero $(1 \mathrm{~g})$ e $1 \mathrm{~mol} \%$ de catalisador foram misturados e agitados à temperatura de reação.

As reações de polimerização foram realizadas em batelada, em microrreatores com fundo cônico de $3 \mathrm{~mL}$ (Supelco), equipados com tampa e septo, e agitadas à temperatura desejada. Após 6 horas, as reações foram dissolvidas em THF e a polimerização foi interrompida com excesso de etil vinil éter e agitação por 30 minutos à temperatura ambiente. $\mathrm{O}$ resíduo foi precipitado em metanol gelado e, então, o polímero foi filtrado para posterior caracterização.

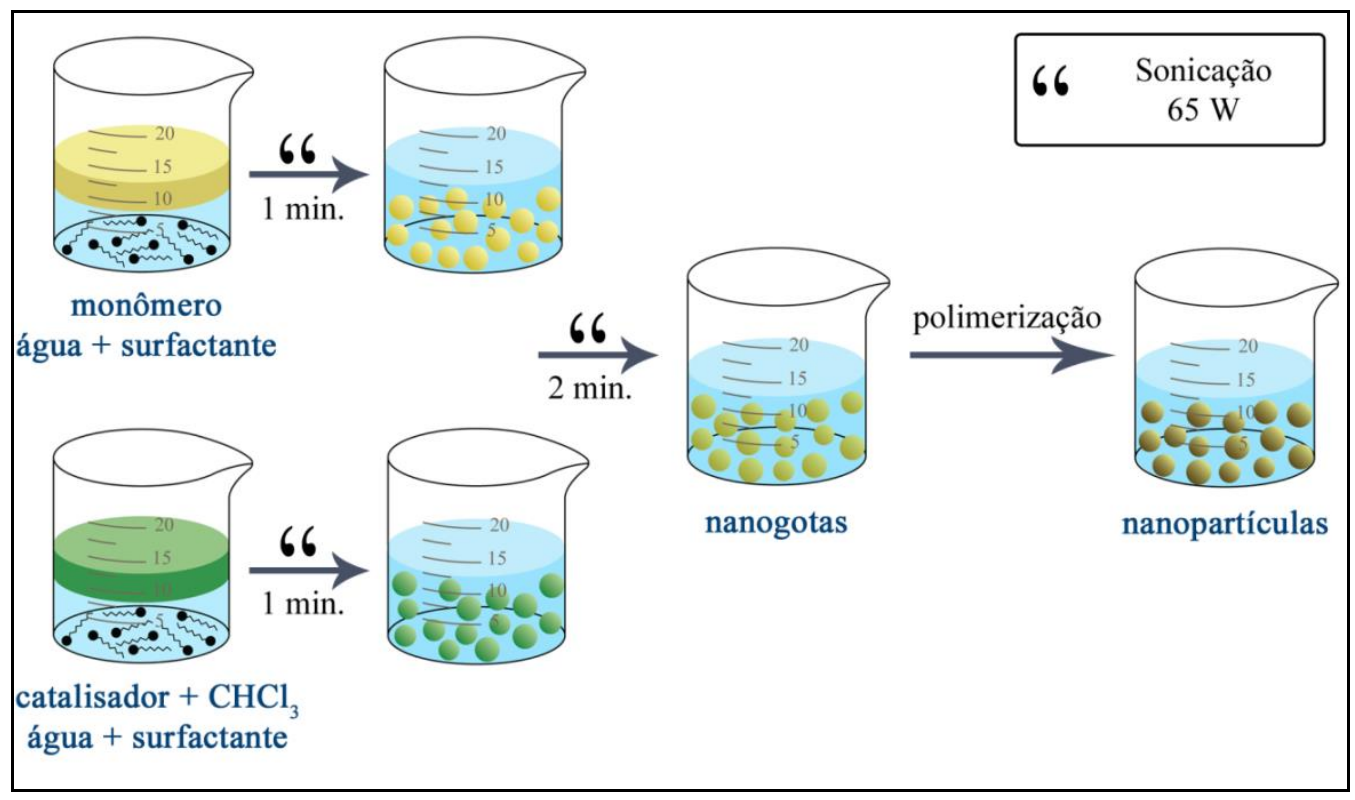

Figura 1 - Esquema simplificado do processo de polimerização em miniemulsão. 


\subsection{Caracterização}

O diâmetro médio (em intensidade) das gotas de monômero e das partículas de polímero foi determinado por Espalhamento Dinâmico de Luz (DLS) utilizando o equipamento Zetasizer Nano S, da Malvern Instruments.

As análises de massa molar foram realizadas pela técnica de cromatografia por permeação em gel (GPC) em um equipamento da marca Shimadzu, modelo LC-20A equipado com injetor automático SIL-20A, pré-coluna PSS SDV $(5 \mu \mathrm{m}, 8 \times 50 \mathrm{~mm})$, coluna principal PSS SDV $10000 \AA$ $(5 \mu \mathrm{m}, 8 \times 300 \mathrm{~mm})$ e detector de índice de refração RID-10A em THF (vazão de $1 \mathrm{~mL} \mathrm{~min}^{-1}$ ) a $50{ }^{\circ} \mathrm{C}$. Para determinar a massa molar das amostras, padrões de PMMA (polimetacrilato de metila), com massas molares ponderais médias variando de 1.1 a $981 \mathrm{kDa}$, foram utilizados.

As análises de ressonância magnética nuclear de ${ }^{1} \mathrm{H}$ e de ${ }^{13} \mathrm{C}$ do monômero sintetizado foram registradas em clorofórmio deuterado $\left(\mathrm{CDCl}_{3}\right)$ em espectrômetros BRUKER AVANCE DPX operando a 300 e $600 \mathrm{MHz}$. Os desvios químicos $(\delta)$ são reportados em partes por milhão em relação ao padrão interno de tetrametilsilano (TMS, $\delta=0,00 \mathrm{ppm}$ ).

A temperatura de fusão cristalina dos polímeros foi determinada utilizando-se um equipamento de Calorimetria Diferencial de Varredura (Differencial Scanning Calorimetry DSC), Mettler Toledo DSC 823, com taxa de aquecimento e resfriamento de $10{ }^{\circ} \mathrm{C} \mathrm{min}{ }^{-1} \mathrm{sob}$ atmosfera de nitrogênio $\left(30 \mathrm{~mL} \mathrm{~min}^{-1}\right)$. As curvas do segundo aquecimento foram registradas.

\section{RESULTADOS E DISCUSSÃO}

Para iniciar a nossa investigação, o monômero $\alpha, \omega$-dieno 1,3-propileno diundeca-10-enoato foi preparado por um procedimento encontrado na literatura (Mutlu et al., 2010) utilizando 1,3propanodiol, que pode ser preparado por fermentação do glicerol, e ácido 10-undecenóico, um derivado comercial de óleo de mamona e uma importante matéria-prima para cosméticos, produtos farmacêuticos e compostos poliméricos. Reações de polimerização ADMET do monômero $100 \%$ renovável (Figura 2) foram realizadas para estudar o efeito do catalisador e do tipo de surfactante a diferentes temperaturas $\left(45\right.$ e $\left.85{ }^{\circ} \mathrm{C}\right)$, após 6 horas de reação, em miniemulsão (Mini) e massa (B) e os resultados são apresentados na Tabela 1.

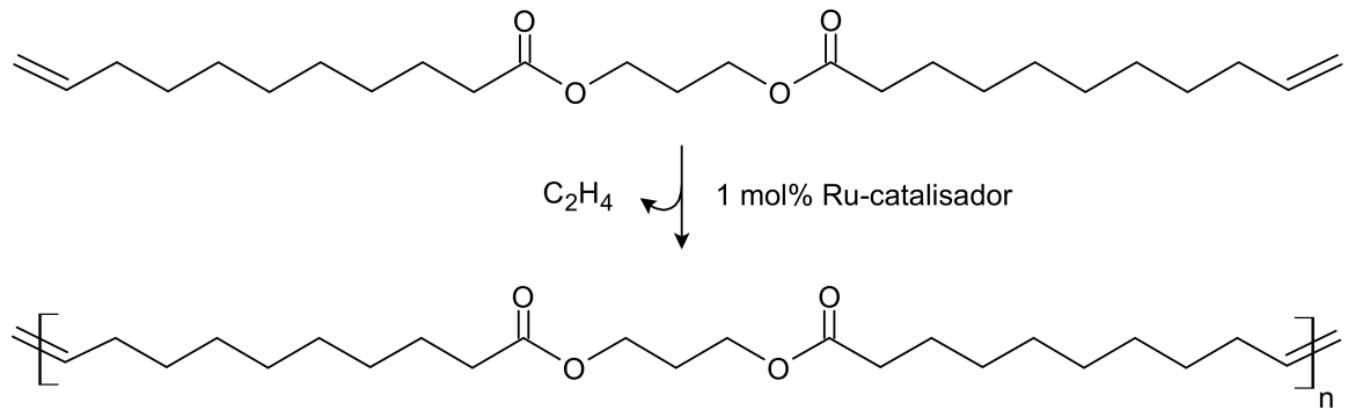

Figura 2 - Polimerização ADMET do monômero 1,3-propileno diundeca-10-enoato. 
Tabela 1 - Formulação e resultados das reações de polimerização ADMET em miniemulsão ${ }^{a}$

\begin{tabular}{cccccccc}
\hline Reação & Cat. & $\boldsymbol{T}\left({ }^{\circ} \boldsymbol{C}\right)$ & Surfactante [wt.\% $]^{c}$ & Dp $(\boldsymbol{n m})$ & $\boldsymbol{P D I}$ & $\boldsymbol{M}_{\boldsymbol{n}}(\boldsymbol{k D a})$ & $\boldsymbol{M}_{\boldsymbol{w}}(\boldsymbol{k D a})$ \\
\hline $\mathbf{1}$ & HG2 & 45 & SDS [0.3\%] & 329 & 0.25 & - & - \\
$\mathbf{2}$ & HG2 & 85 & SDS [0.3\%] & 349 & 0.29 & - & - \\
$\mathbf{3}$ & HG2 & 45 & CTAB [0.3\%] & 156 & 0.12 & 1.0 & 1.5 \\
$\mathbf{4}$ & HG2 & 85 & CTAB [0.3\%] & 192 & 0.16 & 2.2 & 2.6 \\
$\mathbf{5}^{\boldsymbol{b}}$ & HG2 & 85 & - & - & - & 4.5 & 8.6 \\
$\mathbf{6}$ & HG2 & 85 & Lutensol AT80[3\%] & 243 & 0.15 & 2.7 & 4.0 \\
$\mathbf{7}$ & G1 & 85 & CTAB [0.3\%] & 186 & 0.16 & 1.2 & 2.0 \\
$\mathbf{8}$ & G1 & 85 & Lutensol AT80[3\%] & 212 & 0.17 & 1.4 & 2.1 \\
$\mathbf{9}^{\boldsymbol{b}}$ & G1 & 85 & - & - & - & 2.0 & 3.4 \\
$\mathbf{1 0}$ & UM2 & 85 & CTAB [0.3\%] & 169 & 0.14 & 7.2 & 15.1 \\
$\mathbf{1 1}$ & UM2 & 85 & Lutensol AT80[3\%] & 302 & 0.12 & 14.7 & 26.5 \\
$\mathbf{1 2}^{\boldsymbol{a}}$ & $\mathrm{UM} 2$ & 85 & - & - & - & 4.7 & 9.4 \\
\hline
\end{tabular}

a Tipo de catalisador (Cat.), temperatura, tipo de surfactante, diâmetro médio das partículas (Dp), índice de polidispersão (PDI), massa molar média numérica $\left(\mathrm{M}_{\mathrm{n}}\right)$ massa molar média ponderal $\left(\mathrm{M}_{\mathrm{w}}\right)$;

${ }^{\mathrm{b}}$ Reação realizada em massa; ${ }^{\mathrm{c}}[$ ] de surfactante em relação à água.

Todas as reações se apresentaram estáveis e resultaram em tamanhos médios de partícula na faixa de 150 a $350 \mathrm{~nm}$, dependendo do tipo de surfactante utilizado. A concentração dos surfactantes dodecil sulfato de sódio (SDS) e brometo de cetiltrimetilamônio (CTAB) foi mantida em 0,3\% em massa (relativa à água) e a concentração do surfactante não iônico Lutensol AT80 foi mantida em 3\%. A diferença de fração mássica entre os surfactantes é devida à massa molar muito mais alta do Lutensol AT80. Quando comparadas as frações molares dos surfactantes em relação à água, Lutensol e CTAB apresentam frações molares similares, 0,014 e 0,015 mol\%, respectivamente. Embora o SDS tenha apresentado fração molar maior, $0,019 \mathrm{~mol} \%$, a estabilização das gotas de monômero foi menos eficaz, com um diâmetro médio de cerca de $300 \mathrm{~nm}$, e a distribuição de tamanho de partícula muito polidispersa (PDI superior a 0,2). SDS é um surfactante geralmente muito eficaz, mas os resultados mostraram que houve uma interação entre o SDS e o catalisador Hoveyda-Grubbs $2^{\text {a }}$ geração (HG2), diminuindo a eficiência de estabilização do SDS.

Os primeiros experimentos (reações 1 e 2) mostraram que não ocorreu polimerização quando utilizado o catalisador $\mathrm{HG} 2$ na presença do surfactante aniônico SDS em 45 ou $85^{\circ} \mathrm{C}$. Comportamento semelhante foi observado por Pecher e Mecking (2007) quando utilizado o SDS para polimerizar 1,4-dipropoxi-2,5-divinilbenzeno através de reação ADMET em miniemulsão aquosa usando os catalisadores Grubbs de $2^{a}$ geração e HG2, onde não ocorreu polimerização. Também foi reportado por Lynn et al. (1996) que baixas conversões de polinorborneno foram obtidas através de reações ROMP usando G1 como catalisador e o surfactante aniônico SDS. Conversões maiores foram atingidas quando foi utilizado o surfactante catiônico DTAB (brometo de dodeciltrimetilamônio). 


\section{9 a 22 de outubro de 2014 \\ Florianópolis/SC}

Reações utilizando CTAB como surfactante (reações 3 e 4) mostraram que o surfactante catiônico não desativou completamente o catalisador HG2, ao contrário do que ocorreu com o SDS. Entretanto, massas molares mais baixas foram obtidas em comparação à reação em massa (reação 5). Este resultado pode ser atribuído à decomposição parcial do catalisador $\mathrm{HG} 2 \mathrm{em}$ água. $\mathrm{O}$ aumento da temperatura de reação de 45 para $85{ }^{\circ} \mathrm{C}$ resultou em um ligeiro aumento na massa molar do polímero obtido devido ao aumento da atividade do catalisador com o aumento da temperatura.

O efeito do tipo de catalisador (G1, HG2, UM2) em miniemulsão foi investigado sob condições semelhantes em termos de concentração de reagentes na fase orgânica e temperatura (reações 4 , 7 e 10) usando $C T A B$ como surfactante. A distribuição de massa molar dos polímeros foi analisada por GPC (Figura 3a). Os valores de massa molar numérica média, $\mathrm{M}_{\mathrm{n}}$, dos polímeros obtidos seguiram a ordem: UM2 $(\sim 7 \mathrm{kDa})>>\mathrm{HG} 2(\sim 2 \mathrm{kDa})>\mathrm{G} 1(\sim 1 \mathrm{kDa})$. Este resultado está de acordo com alguns trabalhos encontrados na literatura que mostraram que todos os catalisadores Ru-indenilidenos, como o UM2, foram mais robustos sob condições similares de reação quando comparados aos seus catalisadores Ru-benzelidenos homólogos, como G1 e HG2 (Mutlu et al., 2010).

Reações usando o surfactante não iônico Lutensol AT80 com diferentes catalisadores (reações 6,8 e 11) resultaram em polímeros com massas molares mais altas (Figura 3b) quando comparadas às massas molares obtidas nas reações com os mesmos catalisadores usando o surfactante catiônico CTAB (reações 4, 7 e 10; Figura 3a). Este efeito foi mais pronunciado quando comparados os polímeros P10 $\left(\mathrm{M}_{\mathrm{n}}=\sim 7 \mathrm{kDa}\right)$ e P11 $\left(\mathrm{M}_{\mathrm{n}}=\sim 15 \mathrm{kDa}\right)$, obtidos com CTAB e Lutensol, respectivamente, e UM2 como catalisador. A maior reatividade observada nas reações com o surfactante não-iônico indicou que a carga eletrônica dos surfactantes afetou a atividade do catalisador, embora o surfactante catiônico não tenha desativado o catalisador na mesma intensidade que ocorreu com o surfactante aniônico.
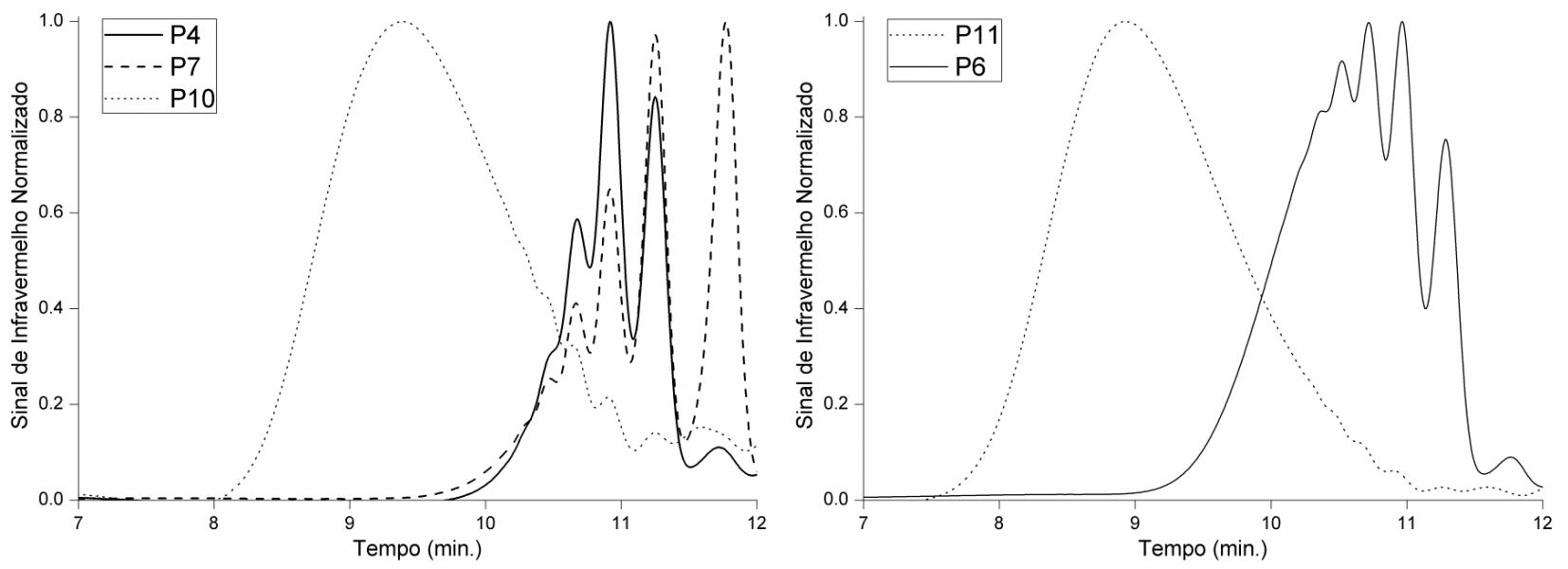

Figura 3 - Distribuição de massa molar dos látices obtidos nas reações de polimerização ADMET em miniemulsão realizadas com: a) HG2 (P4), G1 (P7) e UM2 (P10) como catalisadores e CTAB como surfactante (reações 4, 7 e 10); b) HG2 (P6) e UM2 (P11) como catalisadores e Lutensol AT80 como surfactante (reações 6 e 11). 
Os resultados de DSC (Figura 4) mostraram que o polímero P11 (reação 11), obtido com UM2 e Lutensol AT80, exibiu um pico de $\mathrm{T}_{\mathrm{m}}$ (temperatura de fusão cristalina) bastante estreito em $30,0{ }^{\circ} \mathrm{C}$, indicando uma maior regularidade estrutural em comparação com o polímero P10 (reação 10), obtido com UM2 e CTAB, que apresentou um pico largo de temperatura de fusão provavelmente devido à sua larga distribuição de massa molar contendo picos de polímero de baixa massa molar.

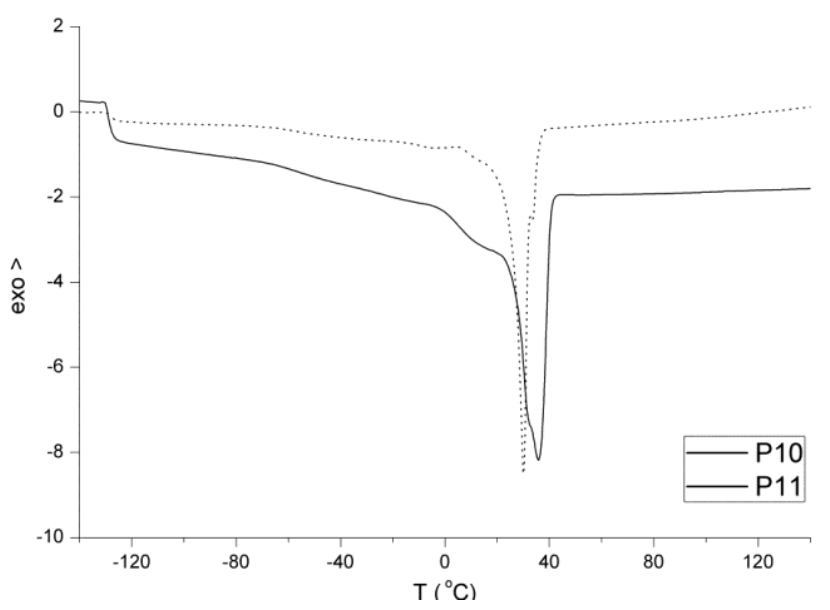

Figura 4 - Curvas de DSC do segundo aquecimento para os polímeros P10 e P11 (reações 10 e 11 ).

A massa molar da reação em miniemulsão utilizando o catalisador UM2 e o Lutensol AT80 como surfactante (reação 11) foi mais do que três vezes maior do que massa molar da reação em massa (reação 12). Aparentemente, a área superficial da fase orgânica muito maior na reação em miniemulsão facilitou a liberação do etileno (formado durante a reação de metátese) das partículas de polímero, promovendo a reação ADMET e atingindo uma massa molar mais alta do que a atingida na reação em massa, uma vez que todas as reações foram realizadas sem qualquer tentativa (tal como vácuo ou fluxo de nitrogênio) de remoção do etileno do meio reacional. Adicionalmente, os resultados mostraram que a combinação do catalisador Umicore M2 e do surfactante não-iônico Lutensol AT80 foi a mais apropriada para as reações ADMET em miniemulsão resultando no polímero com maior massa molar obtido no presente trabalho.

\section{CONCLUSÕES}

Reações ADMET em miniemulsão foram realizadas com sucesso e foram obtidos polímeros com $\mathrm{M}_{\mathrm{n}}$ de até $15 \mathrm{kDa}$ dependendo do catalisador e do surfactante utilizados. O catalisador $\mathrm{Ru}-$ indenilideno Umicore M2 apresentou maior atividade em água quando comparado aos catalisadores Ru-benzelidenos Hoveyda-Grubbs de $2^{a}$ geração e Grubbs de $1^{a}$ geração. Além disso, os resultados mostraram que a atividade dos catalisadores foi significativamente afetada pelo tipo de surfactante (aniônico, catiônico ou não-iônico). A grande área superficial da fase orgânica nas reações em miniemulsão provavelmente promoveu a remoção de etileno do meio reacional, favorecendo a reação ADMET e atingindo polímeros com massas molares maiores do que as reações em massa. Através dos resultados, foi observado que o catalisador Umicore M2 e o surfactante não-iônico Lutensol AT80 formaram a combinação mais adequada para a realização de reações ADMET em miniemulsão. 


\section{REFERÊNCIAS}

ANTONIETTI, M.; LANDFESTER, K. Polyreactions in miniemulsions. Prog. Polym. Sci., v. 27, p. 689-757, 2002.

ASUA, J. M. Miniemulsion polymerization. Prog. Polym. Sci., v. 27, p. 1283-1346, 2002.

CARDOSO, P. B.; ARAÚJO, P. H. H.; SAYER, C. Encapsulation of jojoba and andiroba oils by miniemulsion polymerization. Effect on molar mass distribution. Macromol. Symp., v. 324, p. 114123, 2013.

CARDOSO, P. B.; MUSYANOVYCH, A.; LANDFESTER, K.; SAYER, C.; ARAÚJO, P. H. H. ADMET reactions in miniemulsion. J. Polym. Sci. Pol. Chem., v. 52, p. 1300-1305, 2014.

LANDFESTER, K. Miniemulsion polymerization and the structure of polymer and hybrid nanoparticles. Angew. Chem. Int. Edit., v. 48, p. 4488-4507, 2009.

LYNN, D. M.; KANAOKA, S.; GRUBBS, R. H. Living ring-opening metathesis polymerization in aqueous media catalyzed by well-defined ruthenium carbene complexes. J. Am. Chem. Soc., v. 118, p. 784-790, 1996.

MEIER, M. A. R.; METZGER, J. O.; SCHUBERT, U. S. Plant oil renewable resources as green alternatives in polymer science. Chem. Soc. Rev., v. 36, p. 1788-1802, 2007.

MONTERO DE ESPINOSA, L.; MEIER, M. A. R. Plant oils: The perfect renewable resource for polymer science?! Eur. Polym. J., v. 47, p. 837-852, 2011.

MUTLU, H.; MONTERO DE ESPINOSA, L.; TÜRÜNÇ, O.; MEIER, M. A. R. About the activity and selectivity of less well-known metathesis catalysts during ADMET polymerizations. Beilstein J. Org. Chem., v. 6, p. 1149-1158, 2010.

PECHER, J.; MECKING, S. Nanoparticles from step-growth coordination Polymerization. Macromolecules, v. 40, p. 7733-7735, 2007.

PECHER, J.; MECKING, S. Nanoparticles of conjugated polymers. Chem. Rev., v. 110, p. 62606279, 2010.

STAUDT, T.; MACHADO, T. O.; VOGEL, N.; WEISS, C. K.; ARAÚJO, P. H. H.; SAYER, C.; LANDFESTER, K. Magnetic polymer/nickel hybrid nanoparticles via miniemulsion polymerization. Macromol. Chem. Physic., v. 214, p. 2213-2222, 2013. 